\title{
Décentralisation fiscale et croissance des dépenses locales dans les collectivités territoriales décentralisées au Cameroun : cas de la ville de Douala
}

\author{
Nkwenka Nyanda Patrick Geoffroy, PhD \\ Docteur en Sciences économiques, Université de Dschang, Cameroun \\ Temomo Wamba Romuald, \\ Mimché Kouotou Anicet Clément, \\ Doctorant en Sciences de gestion, Université de Dschang, Cameroun
}

\section{Résumé}

La mise en œuvre de la décentralisation soulève l'épineux problème de la mobilisation des ressources pour le développement des collectivités locales. Le budget communal étant tributaire des recettes fiscales, il s'avère nécessaire que la fiscalité locale assure une bonne couverture des dépenses publiques communales. L'objectif de ce papier est de déterminer les contributions de la fiscalité propre, la fiscalité partagée, les subventions et l'emprunt à la croissance des dépenses des collectivités territoriales décentralisées de la ville de Douala. La méthode des moindres carrés ordinaires a permis d'estimer un modèle à effets individuels fixes à partir des données de panel de six collectivités territoriales décentralisées sur la période 2011-2015. Lorsque la fiscalité propre, la fiscalité partagée, les subventions et l'emprunt varient d'une unité, les dépenses locales varient de 104.65\%, $163.69 \%, 13.65 \%$ et $-76,75 \%$, respectivement.

Mots clés : Décentralisation, Recettes, Dépenses, Collectivités térritoriales décentralisées, Cameroun, Douala 


\title{
Fiscal Decentralization and Local Expenditure Growth in the Douala City Council in Cameroon
}

\author{
Nkwenka Nyanda Patrick Geoffroy, PhD
}

Docteur en Sciences économiques, Université de Dschang, Cameroun

Temomo Wamba Romuald, Mimché Kouotou Anicet Clément,

Doctorant en Sciences de gestion, Université de Dschang, Cameroun

\begin{abstract}
The implementation of decentralization raises the problem of the mobilization of the resources for the development of the local communities. The local taxation has to cover the communal public expenditures. The purpose of this paper is to determine the contributions from the local taxation, the shared taxation, the subsidies and the local state bonds to the Douala City Council expenditures. The ordinary least squares method has been used to estimate an individual fixed effect model from the panel data of the six local government making up the Douala City Council over the period going from 2011-2015. When the local taxation, the shared taxation, the subsidies and the local state bonds vary from a unit, the local expenditures vary from $104.65 \%$, $163.69 \%, 13.65 \%$ and $-76.75 \%$, respectively.
\end{abstract}

Keywords: Decentralization; Revenue; Expenditure; Local government; Cameroon; Douala

\section{Introduction}

Dans le cadre de la gouvernance axée sur la Nouvelle Gestion Publique (NGP), le gouvernement local est appelé à faire face aux defis resultant, entre autres, de la decentralisation (Temomo et al., 2019). Une bonne gouvernance financiere des collectivités locales est susceptible de promouvoir le developpement à la base (Temomo et al., 2019).

L'amélioration des conditions de vie des populations à travers le processus de décentralisation ${ }^{2}$ a vu le jour suite au constat d'échec d'une longue période de gestion des affaires publiques par l'Etat avec son lot de réalisations de biens et de services sans réelle contribution des populations

${ }^{2}$ La décentralisation se définit comme le transfert par 1'Etat aux collectivités locales de compétences particulières et de moyens appropriés. 
(Bennett, 1990). Cette forme de gestion s'est soldée par des investissements (en qualité, pérennité et quantité) infructueux (Kamgnia et Touna, 2002). Avec l'avènement de la décentralisation, les populations ont été responsabilisées grâce à l'autonomie de gestion socioéconomique et incitées à prendre des initiatives en s'appuyant essentiellement sur des moyens locaux (humains, matériels et financiers) mis a leur disposition par l'Etat (Yatta, 2009).

La décentralisation est l'une des innovations majeures que connaissent les pays africains depuis les indépendances (Bird et Vaillancourt, 1999). Au Cameroun, elle est perceptible à travers la loi n ${ }^{\circ} 2004 / 017$, portant orientation de la décentralisation, qui stipule en son article 2 alinéa 2 que : "la décentralisation constitue l'axe fondamentale de promotion du développement, de la démocratie et de la bonne gouvernance au niveau local ». Selon le PNUD (2008), l'atteinte de ces différents objectifs passe indubitablement par la disponibilité des ressources financières des Collectivités Territoriales Décentralisées (CTD) ; c'est dans cette optique que la décentralisation fiscale trouve tout son sens.

Selon Yatta (2009), la décentralisation fiscale est la répartition des ressources publiques et l'organisation des rapports financiers entre l'Etat et les collectivités locales. Ce concept englobe la définition d'un champ de ressources propres aux collectivités locales (fiscalité ${ }^{3}$ propre), l'identification d'un champ de ressources partagées entre l'Etat et les collectivités locales (fiscalité partagée), les transferts de ressources de l'Etat aux collectivités locales (subventions) et l'emprunt. Autrement dit, il s'agit d'une véritable décentralisation financière après transfert des pouvoirs aux CTD par l'Etat. La décentralisation fiscale permet alors aux CTD d'être financièrement autonomes vis-à-vis de l'Etat.

D'après Oates (1993), l'autonomie financière des CTD est une voie à privilégier pour le développement local. Elle responsabilise et incite à la transparence dans la gestion. Elle motive les autorités municipales à faire un effort pour élargir l'assiette et améliorer le recouvrement des impôts et taxes (Kom Tchuenté, 1996). Elle permet également de réduire leur dépendance par rapport à l'Etat (Nguini, 1996 ; Weinsou, 2006).

Cependant, la capacité des CTD à promouvoir le développement à la base est limitée par la faiblesse des finances locales et les difficultés financières rencontrées par l'Etat (Finken, 1996 ; Courade, 2000 ; Nkwenka, 2012). Selon Yatta (2009), l'incapacité des CTD à dépenser est à l'origine d'un cercle vicieux dans lequel la pauvreté du cadre de vie entraîne la stagnation, voire la régression relative de l'économie locale : moins une CTD

${ }^{3}$ La fiscalité s'entend comme un système de prélèvement des impôts et taxes autorisés par la loi au profit de l'Etat et des collectivités locales. Au Cameroun, elle relève de la loi à travers les textes suivants : la constitution du 18 janvier 1996 ; le Code Général des Impôts ; la loi n74/23 du 05 décembre 1974 et ses textes modificatifs subséquents. 
dépense, moins elle est capable de convaincre du bien-fondé de l'impôt et moins elle peut mobiliser des ressources. La décentralisation fiscale apparait alors comme un moyen d'améliorer les ressources financières des CTD. Cependant, ladite décentralisation perdrait tout son sens si l'augmentation des finances des CTD ne booste pas les dépenses locales nécessaires à l'amélioration des conditions de vie des populations.

Certaines études ont d'ailleurs montré qu'il existe un lien positif entre les recettes et les dépenses d'une économie locale (Atkinson et Stiglitz, 1980 ; Ahsan et Sahni, 1987). Une pareille étude est opportune dans le contexte camerounais, où la structure 4 de l'économie ne facilite pas la mobilisation des recettes communales ; les CTD font face au détournement des recettes fiscales

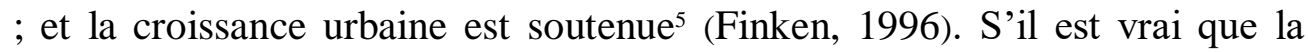
décentralisation fiscale permet le financement du developpement local par la fiscalité propre, la fiscalité partagée, les subventions et l'emprunt ; il reste que la contribution de ces derniers à la croissance des dépenses locales soulève des interrogations, compte tenu de la gouvernance financière des administrations publiques camerounaises, qui s'avère de plus en plus inquiétante au regard des scandales ${ }^{6}$ financiers observés depuis quelques années (Temomo et al., 2019). Par ailleurs, dans la littérature, aucune étude ne s'est penchée sur la contribution de la décentralisation fiscale à la croissance des dépenses des CTD. Pour pallier à cette insuffisance, la problématique de cette étude est la suivante : comment la décentralisation fiscale influence-t-elle les dépenses des CTD ?

Le Cameroun compte plus de 350 collectivités locales. Toutefois, cette étude s'intéresse essentiellement aux six CTD de la ville de Douala, non seulement parce qu'il s'agit d'une étude de cas, mais aussi à cause des caractéristiques $^{7}$ socioéconomiques de ladite ville, et de la disponibilité des données exploitables. L'objectif de ce papier est alors de déterminer les contributions de la fiscalité propre, la fiscalité partagée, les subventions et l'emprunt à la croissance des dépenses des CTD de la ville de Douala.

\footnotetext{
${ }^{4}$ Le secteur informel qui représente $40 \%$ du PIB et $80 \%$ du fichier des contribuables, emploie près de $90 \%$ des actifs occupés, mais fournit seulement $1 \%$ des recettes fiscales totales (Nkwenka, 2012).

${ }^{5}$ La population urbaine au Cameroun a plus que doublé en 17 ans et 6 camerounais sur 10 vivent en zone urbaine. Le taux de croissance urbaine oscille entre 6 et $7 \%$. Le taux d'urbanisation était de 37\% en 1987, puis 55\% en 2004 et 56,48\% en 2009 (Nkwenka, 2012). ${ }^{6}$ Par exemple, le Directeur général du Fonds spécial d'Equipement et d'Intervention Intercommunale (FEICOM) au Cameroun a écopé de graves sanctions suites au détournement des deniers publics (centimes additionnels communaux) en 2000.

${ }^{7}$ Douala est la capitale économique et la plus grande ville du Cameroun. Elle compte environ 120 quartiers répartis en six CTD.
} 
Le reste de l'article est organisé comme suit : la section 1 fait une synthèse des travaux empiriques; ensuite, la section 2 présente la démarche méthodologique; et enfin, la section 3 montre les résultats obtenus.

\section{Synthèse des travaux empiriques et schéma conceptuel de recherche}

Après la présentation des résultats de quelques travaux sur la décentralisation fiscale (1.1), un schéma conceptuel de recherche sera élaboré afin de faciliter la vérification empirique de l'hypothèse de l'étude (1.2).

\subsection{Synthèse des travaux empiriques}

Le regain d'intérêt pour la décentralisation provient de la théorie du fédéralisme financier qui est développée pour la première fois par Hayek (1954). Selon ce dernier, le gouvernement local serait mieux placé que le gouvernement central pour procurer plus efficacement des biens publics à ses résidents. Dans cette logique, Bahl et Shyam (1986), Oates (1993), Shah (1994), Bird et Vaillancourt (1999) montrent que la décentralisation fiscale est une mesure nécessaire pour baisser le déficit budgétaire et accroitre l'efficacité du secteur public.

A partir d'un échantillon de pays en développement (Amérique latine et Asie), Fukasaku et De Mello (1993) ont montré que la décentralisation fiscale a un impact positif sur la situation budgétaire lorsque les dépenses locales sont entièrement financées par les ressources locales.

De même, Atsushi (2004), à partir d'un modèle de croissance endogène et de données de la période 1997-2001, montre l'impact positif de la décentralisation fiscale sur la croissance économique. En effet, plus la part des dépenses publiques mises en œuvre par les CTD est importante, plus la croissance du PIB par habitant est élevée.

Toutefois, Xie et al. (1999) et Zang et Zou (1999) montrent un lien négatif important entre la décentralisation fiscale et la croissance économique. Selon ces derniers, le transfert de responsabilités de dépenses aux CTD n'est pas compensé par une réduction proportionnelle des dépenses de l'Etat. De ce fait, le transfert de ressources aux CTD pourrait avoir privé l'Etat des ressources importantes.

Davoodi et Heng-fu (1997) mettent en lumière une relation négative entre la décentralisation fiscale et la croissance économique. Une augmentation de $10 \%$ du niveau de décentralisation fiscale se traduit par une baisse de $0,7 \%$ du taux de croissance économique pour l'ensemble des pays et de $0,8 \%$ pour les pays en développement. Selon ces derniers, la décentralisation fiscale déprofessionnalise les décisions techniques délicates. Aussi, la décentralisation fiscale se traduit dans ces pays par la non articulation entre les politiques nationales et locales, engendrant ainsi un manque de 
complémentarité et au pire un gaspillage de ressources dans les actions souvent contradictoires.

Selon Ebel et Yilmaz (2002), les décentralisations africaines ont pour caractéristiques communes la faiblesse des ressources financières des collectivités locales et l'inadéquation des arrangements financiers entre l'Etat et les CTD.

La plus part des travaux sur la décentralisation fiscale en Afrique ont été réalisés sur les pays anglophones : c'est notamment le cas des travaux de Smoke (2001) et Chambas (2005). Sur les pays d'Afrique de l'Est et Australe, les travaux ont évalué le partage des ressources entre l'Etat et les CTD au regard des compétences transférées (Kafimbou et Sanou, 2007). Peu de travaux se sont focalisés sur les pays d'Afrique Subsahararienne (Yatta, 2009).

$\mathrm{Au}$ Cameroun, les travaux sur la décentralisation fiscale sont rares (GTZ, 2005). Finken (1996) donne une explication du concept et des paramètres de la décentralisation. Il analyse l'aspect historique de l'institution municipale en décrivant les finances communales. Nkwenka (2012) analyse les différentes causes des déséquilibres financiers entre les communes camerounaises en proposant des solutions pour y remédier. En outre, plusieurs documents de référence à la fois pour les élus locaux et les cadres des administrations locales exposent un ensemble de techniques visant à améliorer la gestion des finances locales en vue de la promotion du développement à la base.

La contribution de la décentralisation fiscale à la croissance des dépenses des CTD a rarement fait l'objet des investigations empiriques, or les résultats d'un pareil travail auraient des implications évidentes pour les politiques et les prévisions budgétaires locales.

\subsection{Schéma conceptuel de recherche}

Plus de $50 \%$ des ressources financières des collectivités locales africaines proviennent des financements apportés par les partenaires extérieurs (Weinsou, 2006). La décentralisation fiscale en Afrique apparait donc comme l'une des problématiques majeures du développement local (Hilhorst et Baltissen, 2005). La mobilisation des ressources financières locales est alors devenue un véritable challenge pour l'ensemble des CTD en quête permanente d'une autonomie financière permettant de réaliser des investissements catalyseurs du développement à la base.

La mobilisation et la gestion efficaces des ressources financières reviennent premièrement à disposer des moyens financiers nécessaires pour réaliser les investissements locaux ; et deuxièmement à gérer efficacement lesdites ressources pour gagner la confiance de la population pour qu'elle contribue davantage au financement du développement local (Keita et al., 2008). 
La mise en œuvre de la décentralisation fiscale laisse donc penser que l'amélioration des recettes des CTD (la fiscalité propre, la fiscalité partagée, les subventions et l'emprunt) favoriserait la croissance des dépenses locales (hypothèse). La vérification empirique de cette hypothèse prendra appui sur le schéma conceptuel de recherche présenté dans la figure 1.

Figure 1 : Schéma conceptuel de recherche

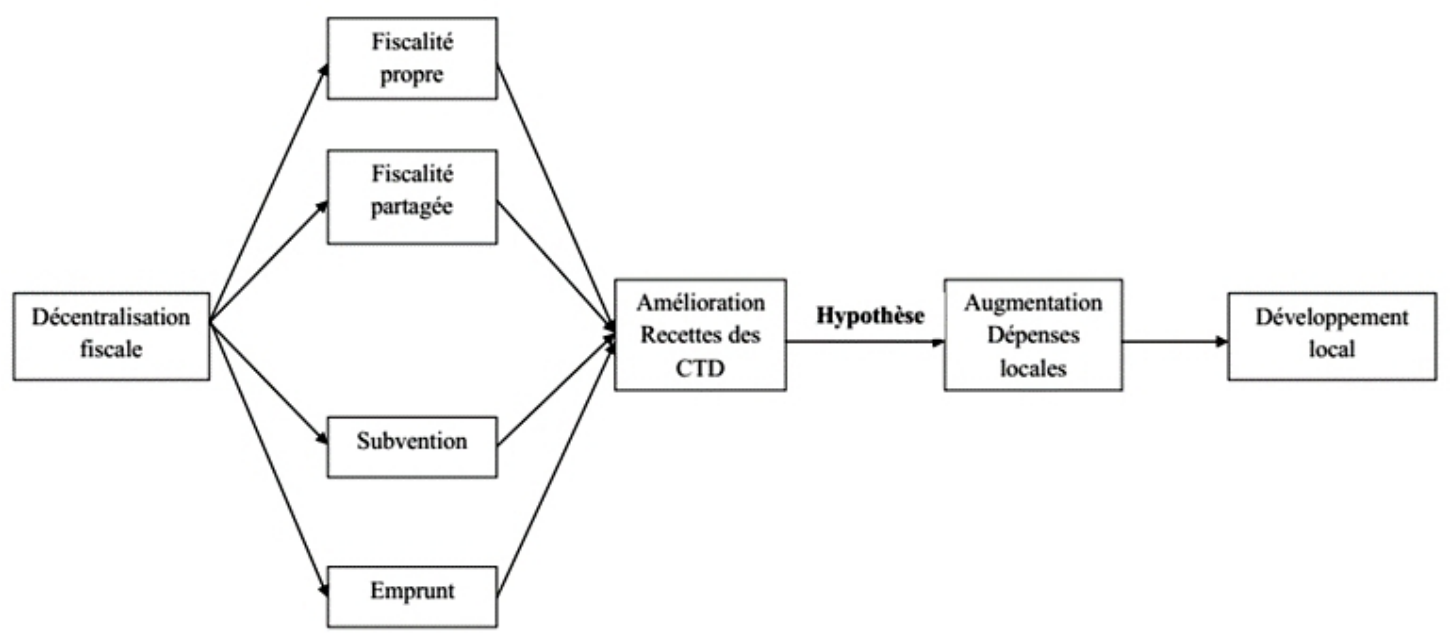

Source : Elaborée par les auteurs, d'après la littérature

(Keita et al., 2008 ; Yatta, 2009)

\section{Démarche méthodologique}

La méthodologie de cette étude est adossée sur la présentation des données (nature et sources), la spécification du modèle économétrique et la description de la méthode d'estimation dudit modèle.

\subsection{Nature et sources de données}

Cette étude porte sur les six CTD de la ville de Douala. Les données exploitées sont quantitatives, annuelles et de source secondaire. Elles proviennent du «Rapport de synthèse de 2015 » de la Communauté Urbaine de Douala et des comptes administratifs de 2011 à 2015 desdites CTD. Le choix de cette période d'étude s'explique par la disponibilité des données et la loi fixant régime financier des CTD promulguée en 2009.

\subsection{Modèle économétrique}

Le modèle économétrique est inspiré des travaux de Cuong (2009). L'équation à estimer se présente ainsi :

$$
D E P_{i t}=\beta_{0}+\beta_{1} F I S P R O_{i t}+\beta_{2} F_{I S P A R_{i t}}+\beta_{3} S U B_{i t}+\beta_{4} E M P_{i t}+\beta_{5} P O P_{i t}+\varepsilon_{i t}(1)
$$


Avec, $\varepsilon_{\text {it }}$ est le terme d'erreur ; i $(\mathrm{i}=1,2,3,4,5,6)$ et $\mathrm{t}(\mathrm{t}=1,2,3,4$, 5) représentent respectivement l'indice de la CTD et celui de l'année ; $\beta_{0}, \beta_{1}$, $\beta_{2}, \beta_{3}, \beta_{4}$ et $\beta_{5}$ sont des paramètres à estimer.

La variable dépendante est DEP (dépenses locales par habitant). Les variables indépendantes sont FISPRO (fiscalité propre), FISPAR (fiscalité partagée), SUB (subvention) et EMP (emprunt). Le tableau 1 récapitule les variables du modèle.

Tableau 1 : Présentation des variables du modèle

\begin{tabular}{|l|c|l|}
\hline \multicolumn{1}{|c|}{ Variables } & Abréviations & \multicolumn{1}{c|}{ Mesures } \\
\hline $\begin{array}{l}\text { Dépenses locales } \\
\text { par habitant }\end{array}$ & DEP & $\begin{array}{l}\text { Somme des dépenses de la CTD / Population } \\
\text { totale de la CTD }\end{array}$ \\
\hline Fiscalité propre & FISPRO & Somme des recettes fiscales propre de la CTD \\
\hline Fiscalité partagée & FISPAR & Somme des recettes fiscales partagées de la CTD \\
\hline Subventions & SUBV & $\begin{array}{l}\text { Somme des subventions reçues de l'Etat et du } \\
\text { FEICOM par les CTD }\end{array}$ \\
\hline Emprunt & EMP & $\begin{array}{l}\text { Somme des emprunts contractés dans le secteur } \\
\text { financier }\end{array}$ \\
\hline $\begin{array}{l}\text { Taille de la } \\
\text { population }\end{array}$ & POP & Somme des individus de la CTD \\
\hline
\end{tabular}

Source : Elaboré par les auteurs, d'après la littérature (Yatta, 2009)

Le tableau 2 présente les signes attendus des variables.

Tableau 2 : Signes attendus des variables explicatives

\begin{tabular}{|c|c|c|c|c|c|}
\hline Variables & FISPRO & FISPAR & SUB & EMP & POP \\
\hline Signes attendus & + & + & + & + & + \\
\hline
\end{tabular}

Sources : Elaboré par les auteurs, d'après la littérature (Cuong, 2009)

Les recettes des CTD (fiscalité propre, fiscalité partagée, subventions et emprunt) représentent les fonds collectés (auprès des individus de la CTD, de l'Etat, du FEICOM et du système financier) pour financer leurs dépenses de fonctionnement et d'investissement. Il en découle qu'une augmentation des recettes devrait entrainer celle des dépenses. Par ailleurs, les recettes des CTD sont collectées auprès de leur population; par conséquent, la hausse de la population entrainerait celle des recettes.

\subsection{Méthode d'estimation}

L'estimation du modèle économétrique de cette étude se fera par la méthode Within/Between. Auparavant, les tests statistiques permettront de valider ledit modèle. 


\subsubsection{Description de la méthode Within/Between}

Ce papier privilégie la prise en compte de l'hétérogénéité du panel à travers l'usage de la méthode d'estimation Within/Between du modèle de panel à effets individuels. Le recours à cette méthode est nécessaire dans la mesure où l'augmentation des dépenses locales s'expliquerait par des facteurs structurels et conjoncturels propres à chaque CTD.

En fixant un individu observé (une CTD), la série chronologique ou la coupe longitudinale le concernant est obtenue. Si c'est la période examinée qui est fixée, une coupe transversale ou instantanée pour l'ensemble des individus est obtenue.

Un modèle à effets individuels fixes est estimé. L'hypothèse de base de ce modèle est que l'hétérogénéité des comportements est modélisée par un effet individuel. Il s'agit donc d'un modèle avec une variable muette individuelle. Par conséquent, ce modèle ressort la variabilité intra-individuelle (estimation Within). Si les perturbations aléatoires croisées satisfont aux hypothèses classiques des Moindres Carrés Ordinaires (MCO), c'est-à-dire, centrées, homoscédastiques, indépendantes et normales, alors l'estimation par les MCO est optimale (Sevestre, 2002).

Ensuite, un modèle à effets individuels aléatoires est estimé. Ici, l'effet individuel n'est plus un paramètre fixe à estimer mais une variable aléatoire non observable. Ce modèle ressort la variabilité inter-individuelle (estimation Between). L'estimation de ce modèle procède par deux étapes : la première consiste à estimer les composantes de la variance de l'aléa et la deuxième consiste à utiliser le résultat de ces estimations pour estimer le modèle par la méthode des Moindres Carrés Généralisés (MCG), la structure de la variance des résidus étant approximativement connue.

\subsubsection{Tests de validation du modèle}

Il s'agit des tests de présence des effets individuels de Fisher, d'hétéroscédasticité de Breusch-Pagan, d'autocorrélation de Wald et d'Hausman.

\section{$>$ Test de présence des effets individuels de Fisher}

Ce test est utilisé pour discriminer le modèle à effet individu et le modèle sans effet individu. Concrètement, l'objectif est de déterminer si le modèle théorique étudié est parfaitement identique pour toutes les CTD, ou au contraire s'il existe des spécificités propres à chaque CTD.

Le logiciel STATA 10 permet de calculer deux statistiques de Fisher. La première teste la significativité conjointe des variables explicatives, tandis que la seconde teste la significativité conjointe des effets individuels fixes introduits. Une p-value inférieure au seuil de signification $\alpha(5 \%)$ traduit la présence d'effets individuels spécifiques. 
$>$ Test d'hétéroscédasticité de Breusch-Pagan

Le test de Breusch-Pagan est utilisé pour détecter la présence d'hétéroscédasticité. L'hypothèse nulle $\left(\mathrm{H}_{0}\right)$ est celle de l'homoscédasticité, tandis que l'hypothèse alternative $\left(\mathrm{H}_{1}\right)$ est celle de l'héteroscedasticité. Si la p-value est inférieure au seuil de signification $\alpha(5 \%)$, on rejette $\mathrm{H}_{0}$ et il y a héteroscedasticité.

\section{$>$ Test d'autocorrélation de Wald}

Le test d'autocorrélation de Wald permet de détecter la présence d'autocorrélation des résidus. Les hypothèses à tester sont :

- $\mathrm{H}_{0}$ : il n'y a pas d'autocorrélation des résidus,

- $\mathrm{H}_{1}$ : il y'a autocorrélation des résidus.

Si la p-value est inférieure au seuil de signification $\alpha(5 \%), \mathrm{H}_{1}$ est acceptée.

\section{$>$ Test d'Hausman}

Le test d'Hausman permet de choisir entre le modèle à effets individuels fixes et le modèle à effets individuels aléatoires. Ce test permet de déterminer si les coefficients des deux estimations (Within et Between) sont statistiquement différents. Les hypothèses à tester sont les suivantes :

- $\mathrm{H}_{0}$ : les coefficients des deux estimations ne sont pas

statistiquement différents,

- $\mathrm{H}_{1}$ : les coefficients des deux estimations sont statistiquement différents.

Si la p-value est supérieure au seuil de signification $\alpha(5 \%)$, on accepte l'hypothèse nulle $\left(\mathrm{H}_{0}\right)$. Le modèle à effets individuels aléatoires est donc le plus approprié. Dans le cas contraire, le modèle à effets individuels fixes est plus adapté.

Ces quatre tests permettent de vérifier si les conditions statistiques d'estimation des paramètres des modèles par la méthode choisie sont remplies.

Tableau 3 : Synthèse des résultats des tests de validation du modèle

\begin{tabular}{|c|c|c|}
\hline Test & Observations (p-value) & Conclusion \\
\hline $\begin{array}{c}\text { Test de détection des } \\
\text { effets individuels }\end{array}$ & Prob $>\mathrm{F}=0,0000<5 \%$ & $\begin{array}{c}\text { Il y a présence des effets } \\
\text { individuels }\end{array}$ \\
\hline Test de Wald & Prob $>$ chi2 $=0,0535>5 \%$ & $\begin{array}{c}\text { Les erreurs ne sont pas } \\
\text { autocorrelés }\end{array}$ \\
\hline Test de Breusch-Pagan & Prob $>\mathrm{F}=0,0000<5 \%$ & $\begin{array}{c}\text { Les erreurs sont } \\
\text { hétéroscédastiques }\end{array}$ \\
\hline Test d'Hausman & Prob $>$ chi2 $=0,0000<5 \%$ & $\begin{array}{c}\text { Le modèle à effets individuels } \\
\text { fixes est approprié }\end{array}$ \\
\hline
\end{tabular}

Source : Elaboré par les auteurs à partir du logiciel STATA 10

(période 2011-2015)

Le tableau 3 montre que le panel est hétérogène puisqu'il y a présence des effets individuels (Prob $>\mathrm{F}=0,0000<5 \%$ ). En plus, le modèle à effets individuels fixes est approprié, au regard du résultat du test d'Hausman. Ce modèle souffre du problème d'hétéroscédasticité des erreurs qui est corrigé 
par la méthode d'Eiker-White (ajout de l'option « robuste » dans STATA 10). Le modèle sera donc estimé par la méthode des MCO.

\section{Résultats}

Le tableau suivant présente les résultats de l'estimation du modèle.

Tableau 4 : Résultats de l'estimation du modèle

\begin{tabular}{|c|c|c|c|c|c|}
\hline Variable & $\begin{array}{c}\text { Coefficient } \\
(\beta)\end{array}$ & Ecart-type & $\mathbf{t}$ & $\mathbf{t}(\mathbf{P}>|\mathbf{t}|)$ & $\begin{array}{c}\text { Dégré de } \\
\text { significativité }\end{array}$ \\
\hline FISPRO & 1,046533 & 0,004876 & 214,60 & 0.000 & $1 \%$ \\
\hline FISPAR & 1,636941 & 0,083111 & 19,70 & 0.000 & $1 \%$ \\
\hline SUB & 0,136529 & 0,002023 & 67,47 & 0.000 & $1 \%$ \\
\hline EMP & $-0,767521$ & 0,027649 & $-27,76$ & 0.000 & $1 \%$ \\
\hline POP & \begin{tabular}{|l|l|} 
& $-0,334051$ \\
\end{tabular} & 0,057390 & $-5,82$ & 0.000 & $1 \%$ \\
\hline $\begin{array}{l}\mathrm{R}^{2} \text { Within } \\
\mathrm{R}^{2} \text { Between } \\
\mathrm{R}^{2} \text { Overall }\end{array}$ & $\begin{array}{l}=0,9998 \\
=0,9989 \\
=0,9989\end{array}$ & \multicolumn{4}{|c|}{$\begin{array}{c}\text { Nombre d'années “ } \mathrm{t} \text { ” }=5 \\
\text { Prob }>\mathrm{F}=0,0000\end{array}$} \\
\hline
\end{tabular}

Le $\mathrm{R}^{2}$ Between $(0,9989)$ montre la contribution des effets fixes au modèle, ce qui signifie que le modèle est expliqué à $99.89 \%$ par les effets fixes. Le $\mathrm{R}^{2}$ Within met en relief la variation intra-individu de la variable dépendante, expliquée par celle des variables explicatives. Dans le modèle à effets individuels fixes, c'est le $\mathrm{R}^{2}$ Within qui est plus approprié puisqu'il est de 0.9998 , ce qui signifie que la variation intra-individu de la variable dépendante est expliquée à $99.98 \%$ par celle des variables explicatives. Le modèle est alors globalement acceptable. De plus, le test global de Fisher révèle une p-value (Prob $>\mathrm{F}=0,0000$ ) inférieure au seuil de signification $\alpha$ $(5 \%)$; c'est-à-dire que le modèle est globalement bien spécifié.

Le test de Student révèle que, pour chaque coefficient, la p-value $(\mathrm{P}>$ $|\mathrm{t}|)$ est inférieure au seuil de signification $\alpha(1 \%)$. Tous les coefficients du modèle sont individuellement significatifs au seuil de $1 \%$. Ceci montre que l'effet respectif des variables indépendantes (FISPAR, FISPRO, SUB, EMP et POP) sur la variable dépendante (DEP) est significatif.

La fiscalité propre (FISPRO), la fiscalité partagée (FISPAR), les subventions (SUB) ont des coefficients positifs. Ceci signifie que, ces variables évoluent dans le même sens que les dépenses locales (DEP). Ainsi, lorsque la fiscalité propre, la fiscalité partagée et les subventions varient d'une unité, les dépenses locales varient dans le même sens de $104.65 \%, 163.69 \%$ et $13.65 \%$, respectivement. Les signes de ces trois variables (FISPAR, FISPRO et SUB) sont conformes aux signes attendus. La fiscalité propre et la fiscalité partagée contribuent le plus à l'expansion des dépenses des CTD. Ceci témoigne à suffisance de l'importance de ces modes de prélèvements fiscaux pour la consolidation du processus de décentralisation fiscale. Les CTD 
devraient donc définir des mesures propices à l'amélioration des recettes fiscales locales.

L'emprunt (EMP) et la taille de la population (POP) ont des coefficients négatifs. Ceci signifie que ces variables n'évoluent pas dans le même sens que les dépenses locales. Ainsi, lorsque l'emprunt et la taille de la population varient d'une unité, les dépenses locales varient dans le sens contraire, respectivement de $-76,75 \%$ et $-33,40 \%$. Les signes de ces deux variables (EMP et POP) ne sont pas conformes aux signes attendus. Ces résultats rejoignent ceux de Cuong (2009), Moussa et Iboudo (2007), Davoodi et Heng-fu (1997) et Fukasaku et De Mello (2002).

Le signe de EMP peut s'expliquer d'une part à travers l'action individuelle des CTD de la ville de Douala, et d'autre part, à travers l'action du temps. L'action individuelle est liée au fait que, la pratique de l'emprunt dans le financement de la décentralisation est plus développée dans certaines CTD que dans d'autres, qui pour la majorité ne disposent pas du niveau d'expertise suffisant pour réaliser ce type d'opération. A Douala ${ }^{8}$, seulement la Communauté Urbaine dispose de l'habilitation à emprunter sur le marché financier. L'effet individuel peut aussi s'expliquer par la faiblesse de l'épargne des CTD. Ladite faiblesse serait expliquée par les insuffisances de gestion locale, mais aussi par la dépendance financière des CTD vis-à-vis de l'Etat. De même, le principe d'unicité de caisse (prévarication des ressources des CTD dans un contexte de crise de finances publiques de l'Etat) compromet l'emprunt des CTD. Elle peut mettre une CTD dans l'impossibilité d'honorer à un remboursement de prêt. L'action du temps est liée au fait que, la pratique de l'emprunt n'est pas régulière dans le temps (l'emprunt n'est pas contractée chaque année par les CTD).

Le signe de POP peut s'expliquer par le fait qu'une partie significative de la population (secteur informel) échappe au paiement de l'impôt. Avec l'expansion du chômage, les jeunes se tournent de plus en plus vers le secteur informel. Une autre justification se trouverait dans le fait que les individus payent les impôts sans recevoir de contrepartie en matière d'investissements publics, à cause des détournements de fonds par les autorités communales. Ceci est aussi dû au dysfonctionnement des communes, et au retrait des fonds aux CTD par l'Etat. Tout ceci freine l'augmentation des dépenses locales, pendant que la population augmente.

\footnotetext{
${ }^{8}$ Un exemple est donné par celle-ci en 2005 : en effet, suite à l'autorisation du Ministère en charge de la tutelle des collectivités locales et du Ministère de l'économie et des finances, la Communauté Urbaine de Douala a réalisé des émissions obligataires sur le marché des capitaux camerounais, la Douala Stock Exchange, en deux temps. La première pour un montant de 13,5 millions de dollars et la seconde pour un montant de 5,5 millions de dollars.
} 


\section{Conclusion}

La problématique du financement du développement local par la décentralisation fiscale a suscité l'intérêt de cette étude qui s'est attelée à évaluer les contributions de la fiscalité propre, la fiscalité partagée, les subventions et l'emprunt à la croissance des dépenses des CTD de la ville de Douala. Alors que la plupart des travaux empiriques antérieurs se sont limités à déterminer l'effet de la décentralisation fiscale sur la croissance économique (macro-analyse), la présente étude marque son empreinte par une mésoanalyse des finances locales décentralisées. Ladite analyse montre l'effet positif de la fiscalité locale (propre et partagée) et des subventions sur les dépenses communales. De ce fait, les CTD gagneraient à s'investir davantage à l'amélioration de leurs recettes budgétaires par la définition d'une politique de recouvrement fiscal optimal. L'Etat pourrait aussi assurer la disponibilité permanente des ressources financières communales en supprimant le principe d'unicité de caisse (toutes les ressources financières de l'Etat et des CTD sont centralisées dans les caisses de l'Etat). Par ailleurs, les signes négatifs de l'emprunt et la taille de la population interpellent le gouvernement local, sous l'assistance et le contrôle de l'Etat, à se doter de moyens humains, matériels et financiers permettant de réaliser les opérations d'emprunt sur le marché financier. Il est toutefois important de relever que cette étude n'a pas évalué la contribution de la fiscalité locale aux recettes budgétaires des CTD, ce qui aurait permis de mieux apprécier leur niveau d'autonomie financière. Cette préoccupation pourrait être prise en compte dans les travaux empiriques futurs.

\section{References:}

1. Ahsan, S. M. et Sahni, B. S., (1987), « la relation entre les dépenses et les recettes publiques dans une économie régionale : le Quebec, 195582 », L’Actualité économique, vol. 63, n²4, pp. 295-310.

2. Atkinson, A. B. et Stiglitz, J. E., (1980), Lectures on Public Economics, New York, McGraw-Hill.

3. Atsushi I. (2004), Decentralization and economic growth revisited: An empirical note, JBICI Working paper, JBICI Institute.

4. Bahl, Roy W. et Shyam, N., (1986), « Public Expenditure Decentralization in Developing Economies », Government and Policy, n4, pp. 405-18.

5. Bennett, R. J., (1990), Decentralization, Local Governments and Markets (Oxford: Clarendon Press).

6. Bird, R. M. et Vaillancourt, F., (1999), «Fiscal decentralization in Developing Countries: an overview », in Bird, R. M. et Vaillancourt, F., (eds), Fiscal decentralization in Developing Countries, Cambridge. Cambridge University Press, pp. 201-221. 
7. Chambas, G., (2005), Afrique du sud du Sahara: mobiliser des ressources fiscales pour le développement, Paris, Economica.

8. Courade, G., (2000), Le désarroi camerounais, Karthala, Paris.

9. Cuong, V. S., (2009), La politique fiscale et le développement $d u$ Vietnam au cours de la transition, Thèse de doctorat es sciences économiques, Université de Paris I Panthéon- Sorbonne.

10. Davoodi, H. et Heng-fu, Z., (1997), « Fiscal Decentralization and Economic Growth A Cross-Country Study », Journal of Urban Economics, $\mathrm{n}^{\circ} 43$, pp. 244-257.

11. Ebel, R. et Yilmaz S., (2002), Le concept de décentralisation fiscale et survol mondial, Symposium international sur le déséquilibre fiscal, Rapport, Annexe 3, Commission sur le déséquilibre fiscal, Québec.

12. Finken, M., (1996), Communes et gestion communale au Cameroun, Presses du groupe ST François, Yaoundé, 348 pages.

13. Fukasaku, et De Mello (1993), Fiscal decentralization and intergovernmental relation in transition economies, Policy Research Working Paper-WPS1122: Washington-WB.

14. Keita, A. et al., (2008), Accroitre les ressources financières des communes : pratiques émergentes en Afrique de l'Ouest et du Centre, sous la direction de G. Baltissen et T. Hilhorst, Amsterdam : KIT.

15. GTZ (2005), Finances communales au Cameroun; Politiques fiscales, fonds de financement, gestion des finances et mobilisation des ressources (Rapport du séminaire thématique DECNET).

16. Hilhorst, T. et Baltissen, G., (2005), Financer la décentralisation rurale; taxes et impôts à l'échelle locale au Bénin, Burkina Faso et Mali. Bulletin 357.

17. Kafimbou, H. et Sanou, M., (2007), Décentralisation et réduction de la pauvreté au Burkina-Faso : quelles stratégies ?, CEDRES, Ouagadougou.

18. Kamgnia, B. et Touna, M., (2002), Le comportement de l'investissement privé au Cameroun : un resserrement de la contrainte financière?, Yaoundé.

19. Kom, T., (1996), Développement communal et gestion urbaine au Cameroun: les enjeux de la gestion municipale dans un système décentralisé, Edition Clé, Yaoundé.

20. Moussa, L. L. et Iboudo E., (2007), Décentralisation en Afrique de l'ouest et du centre, apprendre des expériences locales et intersectorielle : études de cas du Ghana et du Mali.

21.Nguini, O., (1996), L'Etat et les milieux d'affaire au Cameroun: autoritarisme, ajustement au marché et démocratie (1975-1996), CEAN-IEP de Bordeaux et Grap. 
22. Nkwenka, N. P. G., (2012), La décentralisation fiscale comme facteur de développement local au Cameroun: cas de la ville de Douala, Thèse de master en sciences économiques, Université de Dschang, Cameroun.

23. Oates, W., (1993), «Fiscal decentralization and economic development», National Tax Journal, vol. 46, n 2, pp. 237-243.

24. PNUD (2008), Rapport mondial sur le développement Humain 2007/2008, Economica, Paris.

25. Sevestre, P., (2002), Econométrie des données de panel, Dunod, Paris, France.

26. Smoke, P., (2001), Fiscal Decentralization in Developing countries: A review of current concepts and practice, United Nations Research Institute for social development, Democracy, Governance and Human Rights, program paper $n^{\circ} 2$.

27. Shah (1994), The Reform of Intergovernmental Fiscal Relations In Developing \& Emerging Countries, World Bank Policy Research Working Paper $\mathrm{n}^{\circ} 23$, Banque Mondiale.

28. Temomo, W. R. et al., (2019), «De la comptabilité de caisse à la comptabilité en droit constaté : Quels enjeux pour la gouvernance des Etablissements Publics Administratifs ? », Revue Internationale des Sciences de Gestion, vol. 2, n'2, pp. 34-61.

29. Weinsou, C. G., (2006), Le financement du développement : le cas de la commune de POBE au Bénin, SNV, Porto Novo.

30. Xie, D., Zou, H. et Davoodi, H., (1999), «Fiscal decentralization and Economic Growth in the United States », Journal of Urban Economics, vol. 45, pp. 228-239.

31. Yatta, F. P., (2009), La décentralisation fiscale en Afrique : enjeux et perspectives, Karthala, Paris.

32. Zhang, T. et Zou, H.-F., (1999), "Fiscal Decentralization, Public Spending, and Economic Growth on China », Journal of Public Economics, n67, pp. 221-261. 